\title{
A survey of the views and capabilities of community pharmacists in Western Australia regarding the rescheduling of selected oral antibiotics in a framework of pharmacist prescribing
}

\author{
Fatima Sinkala $^{1}$, Richard Parsons ${ }^{1}$, Bruce Sunderland ${ }^{1}$, Kreshnik Hoti ${ }^{2}$, Petra A Czarniak ${ }^{\text {Corresp. } 1}$ \\ ${ }^{1}$ School of Pharmacy, Curtin University, Perth, Western Australia, Australia \\ 2 Faculty of Medicine, Division of Pharmacy, University of Prishtina, Pristina, Kosovo \\ Corresponding Author: Petra A Czarniak \\ Email address: P.Czarniak@curtin.edu.au
}

Background: Antibiotic misuse in the community contributes to antimicrobial resistance. One way to address this may be by better utilizing community pharmacists' skills in antibiotic prescribing. The aims of this study were to examine the level of support for "down-scheduling" selected antibiotics and to evaluate factors determining the appropriateness of community pharmacist prescribing for a limited range of infections, including their decision to refer to a doctor.

Methods: Self-administered questionnaires, including graded case vignette scenarios simulating real practice, were sent to Western Australian community pharmacists. In addition to descriptive statistics and chi-square testing, a General Estimating Equation (GEE) was used to identify factors associated with appropriateness of therapy and the decision to refer, for each of the seven vignettes.

Results: Of the 240 pharmacists surveyed, 90 (37.5\%) responded, yielding 630 responses to seven different case vignettes. There was more than $60 \%$ respondent support for expanded prescribing (rescheduling) of commonly prescribed antibiotics. Overall 426/630 (67.6\%) of the responses chose to treat the patient while the remaining $204 / 630$ (32.4\%) referred the patient to a doctor. Of those electing to treat, $380 / 426(89.2 \%)$ opted to use oral antibiotics, with 293/380 (77.2\%) treating with an appropriate selection and regimen. The GEE model indicated that pharmacists were more likely to prescribe inappropriately for conditions such as otitis media $(p=0.0060)$ and urinary tract infection in pregnancy ( $p<0.0001$ ) compared to more complex conditions. Over $80 \%$ of all pharmacists would refer the patient to a doctor following no improvement within 3 days, or within 24 hours in the case of community acquired pneumonia. It was more common for younger pharmacists to refer the patient to a doctor $(p=0.0165)$.

Discussion: This study adds further insight into community pharmacy/pharmacist characteristics associated with appropriateness of oral antibiotic selection and the decision to refer to doctors. These findings require consideration in designing pharmacist over-the-counter prescribing models for oral antibiotics. 
5 Fatima Sinkala - BPharm, MPharm. Former MPharm student at School of Pharmacy, Curtin

6 University, Bentley, WA. Tel: +61892667369; Fax: +61892662769. Current: Principle

7 Pharmacist II (Clinical Pharmacy MSc), Letsholathebe II Memorial Hospital, Ministry of Health,

8 Botswana. Tel: +267 6879285, (Fax: +267 6860211)

9 gabsfats@gmail.com

11 Richard Parsons - BSc, MSc, PhD. Senior Lecturer, School of Pharmacy, Curtin University,

12 Bentley, WA. Tel: +61892663691 (Fax: +61892662769).

13 R.Parsons@curtin.edu.au

15 Bruce Sunderland - BPharm, DCC, PhD, Emeritus Professor, School of Pharmacy, Curtin

16 University, Bentley, WA. Tel: +61892667377 (Fax: +61892662769)

17 B.Sunderland@curtin.edu.au

19 Kreshnik Hoti - BPharm, PhD. Adjunct Senior Lecturer, School of Pharmacy, Curtin

20 University, Bentley, WA, Assistant Professor, Faculty of Medicine, Division of Pharmacy,

21 University of Prishtina, Pristina, Kosovo

. Tel: $+61892667366($ Fax: +61892662769$)$

23 kreshnik.hoti@uni-pr.edu 
26 Petra Czarniak - BPharm, MPharm, GradCertDiabEd, PhD, Senior Lecturer, School of

27 Pharmacy, Curtin University, Bentley, WA. Tel: +61892667419 (Fax: +61892662769)

28 P.Czarniak@curtin.edu.au

29 


\section{Abstract}

31 Background: Antibiotic misuse in the community contributes to antimicrobial resistance. One

32 way to address this may be by better utilizing community pharmacists' skills in antibiotic 33 prescribing. The aims of this study were to examine the level of support for "down-scheduling"

34 selected antibiotics and to evaluate factors determining the appropriateness of community 35 pharmacist prescribing for a limited range of infections, including their decision to refer to a 36 doctor.

37 Methods: Self-administered questionnaires, including graded case vignette scenarios simulating 38 real practice, were sent to Western Australian community pharmacists. In addition to descriptive 39 statistics and chi-square testing, a General Estimating Equation (GEE) was used to identify 40 factors associated with appropriateness of therapy and the decision to refer, for each of the seven 41 vignettes.

42 Results: Of the 240 pharmacists surveyed, 90 (37.5\%) responded, yielding 630 responses to seven different case vignettes. There was more than $60 \%$ respondent support for expanded prescribing (rescheduling) of commonly prescribed antibiotics. Overall 426/630 (67.6\%) of the responses chose to treat the patient while the remaining 204/630 (32.4\%) referred the patient to a doctor. Of those electing to treat, 380/426 (89.2\%) opted to use oral antibiotics, with 293/380

$47(77.2 \%)$ treating with an appropriate selection and regimen. The GEE model indicated that 48 pharmacists were more likely to prescribe inappropriately for conditions such as otitis media $49(p=0.0060)$ and urinary tract infection in pregnancy $(p<0.0001)$ compared to more complex

50 conditions. Over $80 \%$ of all pharmacists would refer the patient to a doctor following no

51 improvement within 3 days, or within 24 hours in the case of community acquired pneumonia. It

52 was more common for younger pharmacists to refer the patient to a doctor $(\mathrm{p}=0.0165)$. 
53 Discussion: This study adds further insight into community pharmacy/pharmacist characteristics

54 associated with appropriateness of oral antibiotic selection and the decision to refer to doctors.

55 These findings require consideration in designing pharmacist over-the-counter prescribing 56 models for oral antibiotics. 


\section{Introduction}

59 Over the last twenty five to thirty years, a number of countries including New Zealand (NZ),

60 United States of America (USA), United Kingdom (UK), the Netherlands, Japan and Australia,

61 have reclassified or switched several drugs with an established safety profile from prescription to

62 non-prescription availability. [1, 2]. In Australia, examples of drugs that have been reclassified

63 include emergency hormonal contraception, the oral azole antifungal fluconazole and proton

64 pump inhibitors. [1] In NZ and the UK, the antibiotics trimethoprim and azithromycin

65 respectively, may also be supplied by suitably trained pharmacists. [3, 4] As antibiotic resistance

66 has been declared a global threat by the World Health Organization (WHO) [5], employing

67 pharmacists' knowledge and expertise in the appropriate selection and use of reclassified

68 antibiotics could potentially help reduce the level of inappropriate antibiotic use and therefore

69 resistance. [6] Antimicrobial agents available under strict protocols from suitably qualified

70 pharmacists to maintain antimicrobial stewardship could improve patient access to immediate

71 treatment and reduce the workload of general practitioners (GPs). [4, 6, 7] Widespread

72 inappropriate use of antibiotics in hospitals and the community has led to the development of a

73 specialist antibiotic pharmacist's role in the UK. [8] In a study in the community pharmacy

74 setting, researchers in the USA investigated the use of rapid point-of-care tests by pharmacists to

75 allow clinical decision making so that appropriate treatment could be initiated for patients with

76 influenza or group A Streptococcus (GAS) pharyngitis. Researchers reported that this innovative

77 physician-pharmacist disease management program produced positive progress toward reducing

78 the inappropriate use of antibiotics. [9] 
80 Pharmacists are reported to adhere to prescribing guidelines [10] and improve patients' access to

81 medicines. [11] In Australia, the current framework administering access to medicines includes

82 “down-scheduling” and selected medicines previously restricted to 'Prescription only' or

83 'Schedule 4 (S4)' have been reclassified as 'Pharmacist only' or 'Schedule 3 (S3)' medicines,

84 thereby allowing them to be provided over-the-counter (OTC).[2] It is a requirement in Australia

85 that S3 medicines are provided by a pharmacist or under the direct supervision of a 86 pharmacist.[12] In 2010, chloramphenicol ophthalmic products were reclassified from S4 to S3

87 thereby expanding the options and capability of Australian pharmacists treating acute bacterial 88 conjunctivitis and providing improved community access to an effective antibiotic treatment.

89 Similar rescheduling decisions had been made in the UK and New Zealand in previous years.

$90[13]$

92 The roles of community and hospital pharmacists have been extended to include prescribing, in 93 several countries including Canada $[14,15]$, the USA [16], NZ and the UK, where pharmacists 94 are working within various prescribing models, including: collaborative, supplementary and 95 independent pharmacist prescribing.[17] In Australia, expanding the pharmacist's prescribing 96 role is still under review. [18-22] Currently, a range of Schedule 2 (S2) drugs which are only 97 available from pharmacies (Pharmacy only) and S3 drugs which require the direct involvement 98 of a pharmacist (Pharmacist only), are available from community pharmacies for minor or self99 limiting conditions. [19, 23, 24]. A number of protocols are in place on selected 'Pharmacist 100 only' medicines such as prescribing emergency contraception. [12] Access to selected antibiotics 101 via a protocol could enable community pharmacists to effectively treat a range of infections. [19, $10223,24]$ 
104 There are additional factors in the health system in Australia that influence patient access to 105 antibiotics. A repeat prescribing model is part of the Pharmaceutical Benefits Scheme in 106 Australia, whereby a medical practitioner can order repeats for antibiotics initially prescribed by 107 them. The repeat antibiotic prescription is intended to be obtained in case the patient/ client does 108 not adequately improve following the initial course of antibiotics, or a relapse of the same 109 symptoms subsequently occurs. These repeat prescriptions are valid within a period of 12 months 110 following the initial prescription. [25] This may lead to misuse of antibiotics in patients/ clients

111 with repeat prescriptions that choose to self-diagnose and self-manage their symptoms. To

112 address this issue, expanding pharmacists' role in antibiotic prescribing has been suggested. [25]

114 Given that much of the existing literature has explored pharmacists' views on expanding their

115 prescribing role $[17,19,21]$ and support has been given for pharmacist prescribing for a limited 116 range of infections, there is a need for research aimed at assessing pharmacists' perceptions 117 when confronted with various real life scenarios of antibiotic prescribing. [17, 19-21, 26] This

118 would provide valuable insight to policymakers in relation to designing a model of pharmacist

119 prescribing of antibiotics for a limited range of infections in the community setting. This study 120 therefore aims to explore factors determining the appropriateness of community pharmacist 121 prescribing for a limited range of infections, including their decision to refer to a doctor and 122 examine the level of support for "down-scheduling" selected antibiotics.

123

\section{Materials and Methods}


125 This cross-sectional quantitative study involved a postal survey of practising rural and

126 metropolitan community pharmacists in Western Australia (WA). Questions included seven case

127 vignettes which were used due to their ability to simulate key features of a range of 'real-life'

128 scenarios. [27, 28] They were chosen because they carried the external validity strengths of

129 quantitative-survey based research as well as the internal validity strengths of experimental

130 methods. [27, 28] A review of the literature informed the design of the questionnaire [13, 27,

131 28], while the medical conditions shown by scenarios in the vignettes were based on literature

132 and by using the Australian Therapeutic Guidelines (ATG) for antibiotics. [27, 29] The

133 questionnaire and vignettes, which were developed by the researchers, were piloted by six

134 community pharmacists, some with extensive antibiotic experience, for face and content validity

135 and their feedback was used to improve the questionnaire and vignettes.

136

137 The sections of the questionnaire were: (A) demographic information; (B) statements of views

138 on expanding the pharmacist's role in prescribing antibiotics and Section (C) included the case

139 vignettes.

\section{Case vignettes}

142 The case vignettes consisted of seven scenarios and the respondents were asked for their

143 preferred treatment option, under the hypothetical assumption that they were permitted to

144 prescribe oral antibiotics. The design of the case vignettes was such that the final diagnosis was

145 evident. The scenarios included consideration to some of the key features of case-vignette design

146 i.e. experimental aspect (various antibiotic based scenarios and their effect on respondent's

147 choice of treatment); controlled aspect (i.e. same pharmacists responding to different scenarios) 
148 and contextual aspect as demonstrated by variability within each of the scenarios allowing for the

149 verisimilitude of the scenario. [27] The respondents were asked to select an option from: refer to

150 a GP, treat with oral antibiotics (from a list), or choose a different treatment regimen. The list

151 included antibiotic regimens recommended in the ATG Antibiotics. [27, 29] The vignettes were

152 graded according to disease complexity. In cases where the selected management option was not

153 to refer to a GP, the respondent was asked what action they would take if there was no

154 improvement following 24 hours (community acquired pneumonia (CAP) or 3 days (other 155 vignettes).

156

157 Questionnaire distribution

158 The sampling frame was the list of 434 metropolitan and 164 regional community pharmacies

159 available from The Pharmacy Registration Authority of WA. A 40\% sample was randomly

160 selected using a web based randomizer. Hospital pharmacies in WA were excluded. The final

161 questionnaire was distributed to 66 regional and 174 metropolitan community pharmacies in WA

162 in March 2015 and coded to be able to identify non-respondents. A cover letter explaining the

163 objectives and importance of the study was addressed to the 'manager/proprietor' and enclosed

164 with the questionnaires, information sheet and a reply paid envelope. Reminder letters

165 accompanied by additional questionnaires and reply paid envelopes were sent to non-responders 166 in April 2015.

\section{Sample size determination}

169 A sample size of 96 was considered the minimum necessary to conduct the inferential statistics

170 to identify independent variables exhibiting a moderate effect size, with $80 \%$ power and using 
$171 \alpha=0.05[30]$. With an anticipated $40 \%$ response rate based on previous studies [13], 240

172 pharmacies were invited to participate in the study, with the numbers of metropolitan and

173 regional pharmacies in line with the proportions of these in the sample frame (metropolitan

174 community pharmacies made up $72.5 \%$ and regional community pharmacies $27.5 \%$ ).

175

176

177 Data analysis

178 Descriptive statistics (frequencies and percentages) were used to summarize the demographic

179 profile of participants, and their responses. Responses on a five point Likert scale (i.e. strongly

180 agree, agree, neutral, disagree and strongly disagree) were collapsed to a three point Likert scale

181 (agree, neutral and disagree), for the purpose of analysis.

182

\section{Analysis of level of support for expanded prescribing}

184 A composite score representing the respondents' overall attitude towards down-scheduling was obtained as the mean of the first seven statements assessing different aspects of the respondents'

186 support for the down scheduling. The remaining two statements (relating to the design of the

187 pharmacy, and whether OTC oral antibiotics would increase resistance to antibiotics) were not

188 considered to be either supportive or unsupportive of down-scheduling, and therefore not

189 included in the calculation of the overall attitude score. Being a mean of responses on a 1-5

190 scale, the composite score could be interpreted on a similar scale. Therefore, the overall

191 response was classified as "in agreement" if the composite score was between 1 and 2

192 (inclusive), and classified as "not in agreement" otherwise. If the composite score was "in

193 agreement", then the respondent was classified as "supporting rescheduling", and not in support 
194 of rescheduling if the score was "not in agreement". This variable was the primary outcome for

195 the analyses. A logistic regression model was used to identify any demographic variables

196 showing an association with this dichotomous variable. A backwards elimination strategy was

197 used to find the 'optimal' model. In this approach, all the independent variables were initially

198 included in the model, then the least significant was dropped, one at a time, until all variables

199 remaining in the model were significantly associated with the outcome.

200

201 Analysis of vignettes

202 Analysis of factors associated with the choice to refer the patients depicted in the case vignettes

203 to their GP was analysed using a Generalised Estimating Equation (GEE). This model takes into

204 account the correlation between vignette responses made by the same respondent (using an

205 exchangeable correlation matrix structure). Inclusion of the vignette number as a factor in the

206 model allowed a comparison of the referral rates between vignettes. The same model was used to

207 identify factors associated with the appropriate oral antibiotic therapy selected for each vignette,

208 except that cases where the respondent elected to refer straight to the GP were excluded from this

209 analysis. Similarly to the logistic regression model above, a backward elimination strategy was

210 used when fitting the GEE model. The final results are expressed as adjusted odds ratios, their

$21195 \%$ confidence intervals, and p-values.

212

213 A p-value $<0.05$ indicated a statistically significant association. The statistical analyses were

214 performed using the $\mathrm{SAS}^{\odot}$ version 9.2 software.

215 
216 This study was approved by the Human Research Ethics Committee of Curtin University

217 (Approval Number RDHS-04-15).

218

219 Results

220 Of the 240 questionnaires distributed (66 regional and 174 metropolitan), 90 were returned. Of 221 these, 67/90 (74.4\%) were from metropolitan and 23/90 (25.6\%) regional community 222 pharmacists. The response rates from metropolitan community pharmacists $(67 / 174 ; 38.5 \%)$ and 223 rural community pharmacists $(23 / 66 ; 34.8 \%)$ were similar, leading to an overall response rate of $22437.5 \%$ (90/240). Medium sized (based on turn-over of AUD\$1m to \$2m) community 225 pharmacies were the largest cohort (38/89; 42.7\%) (Table 1). Most pharmacists were in the age 226 categories $31-40$ years $(32 / 89 ; 36.0 \%)$ and $21-30$ years $(31 / 89 ; 34.8 \%)$. It is evident (Table 1$)$ 227 that the overall number of respondents supporting the down-scheduling of selected oral 228 antibiotics (as defined by the "composite was 55.6\% (50/90) which was independent of a wide 229 range of demographic pharmacy and pharmacist characteristics.

\section{Respondents' level of support for an expanded prescribing role for oral antibiotics}

237 With respect to pharmacist's views on "down-scheduling" of selected antibiotics, respondents 238 strongly supported statements regarding: the use of their skills and knowledge $(70 / 90 ; 77.8 \%)$, 
239 recognition by pharmacy clients $(72 / 89 ; 80.9 \%)$ and treating of patients in a timely manner

$240 \quad(72 / 90 ; 80.0 \%)$, as illustrated in Figure 1.

241

242

$<$ Insert Figure 1 here $>$

243

244 Results from fitting the logistic regression model showed that no variables were significantly 245 associated with the attitude towards rescheduling. The results indicate that approximately half of 246 the respondents favoured down scheduling regardless of any demographic variables such as their 247 gender, age, or experience. This supports the univariate associations shown in Table 1. A total of $24850 / 89$ pharmacists (i.e. $56.2 \%$ ) estimated that on average 10 or more patients per week would 249 seek advice for conditions where the pharmacist's best option would be to prescribe oral 250 antibiotics, suggesting that they face these situations on daily basis.

251 The two statements which were excluded from calculation of the 'level of support for down252 scheduling' score are included in Figure 1. These show that the current layout of $69 / 90(76.7 \%)$ 253 of pharmacies would be conducive to diagnosis of infections and prescribing of antibiotics. Only $2549 / 90(10 \%)$ of respondents disagreed with the statement that provision of OTC oral antibiotics 255 could increase resistance to antibiotics, with the majority $(65 / 90 ; 72.2 \%)$ agreeing with this 256 statement and the remaining 16/90 (17.8\%) giving a neutral response.

258 Respondents' level of support for community pharmacist prescribing selected oral 259 antibiotics

260 More than 60\% of respondents' (Figure 2) supported expanded prescribing of 261 phenoxymethylpenicillin (56/90), amoxicillin with clavulanic acid (55/88), flucloxacillin 
262 (61/88), cefalexin (64/90), amoxicillin (66/90) with 70/90 (77.9\%) supporting trimethoprim.

263 Few respondents supported rifampicin $(2 / 87 ; 2.3 \%)$.

$264 \quad<$ Insert Figure 2 here $>$

265

266 Case vignette scenarios

267

268 A total of 630 vignette responses were received from the 90 respondents ( 7 vignettes per 269 questionnaire). Overall, responses to $426 / 630(67.6 \%)$ of the vignettes were to treat the patient at 270 presentation compared to the remaining 204/630 (32.4\%) where referral to the GP was the 271 preferred option. Of those who opted to treat, 380/426 (89.2\%) chose to prescribe an oral 272 antibiotic, with $334 / 380(87.9 \%)$ of them selecting an appropriate antibiotic regimen. The 273 decision to immediately treat was $80 / 90(88.9 \%)$ for tonsillitis and $77 / 90(85.6 \%)$ for otitis 274 media. The level of immediate treatment was much lower for acute pyelonephritis 44/90 275 (48.9\%). The GEE model to identify factors associated with the decision to refer the patient to 276 their GP was based on all 630 responses to the case vignettes. Due to the large number of 277 variables in the model, only pairwise interaction terms which would be expected to be correlated 278 with each other were assessed for statistical significance (none was eventually included). Table 2 shows the full results of the GEE analysis. These data show that referral rates were similar for acute pyelonephritis, chlamydial urethritis and urinary tract infections (UTI) in pregnancy. 
288 The difference in choosing to refer between pharmacists of different gender was small (male $28933.5 \%$ vs female $31.2 \%$ ) but statistically significant $(p=0.0198)$, with male pharmacists more 290 likely to refer compared to female pharmacists. In addition, the older respondents were less 291 likely to refer patients $(p=0.0165)$. Respondents from small turnover pharmacies $(p=0.0122)$ 292 were more likely to refer than those from large turnover pharmacies; those who expected only a 293 low number of patients to be seeking advice were more likely to refer, pharmacists with fewer 294 patients treated at their pharmacy where oral antibiotics would be beneficial $(<4$ per week, $295 \mathrm{p}=0.0205)$ and those respondents who were generally not in favour of expanding pharmacists 296 prescribing role in antibiotics were also more likely to refer (Table 2).

297

298

300

301

309 The reference category for comparing the different vignettes was the case of acute 310 pyelonephritis, which had the highest rate of appropriate antibiotic use $(95.5 \%)$. Compared with 
311 this group, antibiotics for otitis media $(p=0.0060)$, UTI in pregnancy $(p<0.0001)$ and CAP

$312(p=0.0283)$ were significantly less appropriately prescribed. The appropriateness of prescribing

313 for tonsillitis $(p=0.5146)$, chlamydial urethritis $(p=0.1337)$ and mild early cellulitis $(p=0.8528)$

314 were similarly appropriate to that for acute pyelonephritis (Figure 3)

315

316 Older respondents ( $>50$ years) were less likely to prescribe appropriately ( $70.8 \%$ vs $78.9 \%$;

$317 p=0.02$ ). Similarly, respondents who were working in the role of a consultant pharmacist (only 3

318 respondents, responding to 13 vignettes), were less likely to prescribe appropriately compared to

319 respondents holding other positions in the pharmacy (69.2\% vs $77.5 \% ; p=0.0068)$.

320

$321<$ Insert Figure 3 here $>$

322

\section{b) Respondents' decision making following no improvement on initial therapy}

324

Following three days of no improvement on initial therapy or 24 hours for CAP (excluding immediate referrals to GP), most respondents would refer the patient to a GP for all vignettes (Figure 4) compared to selecting a different antibiotic, increasing the dose of the current antibiotic, using some alternative treatment or 'other'. For those who did not elect to refer to GP at this later stage, the most common choice was to select another antibiotic, in particular for otitis media $(\mathrm{n}=12 / 14 ; 85.7 \%)$, CAP $(\mathrm{n}=6 / 11 ; 54.5 \%)$ and UTI in pregnancy $(\mathrm{n}=3 / 3 ; 100 \%)$ compared to other vignettes. In the case of CAP, respondents were asked to select therapy after 24 hours of

331 no improvement instead of 3 days. [29]

$333<$ Insert Figure 4 here $>$ 


\section{Discussion}

338 In the hypothetical situation that community pharmacists were permitted to prescribe OTC a range of antibiotics, this study reports their intended antibiotic prescribing behaviour when faced with scenarios simulating real practice. This study therefore provides a detailed insight into the

341 appropriateness of their choice of prescribing for a graded range of infections, and identifies

342 factors associated with appropriate prescribing. In addition it identifies medical conditions where

343 the pharmacist would generally choose to refer the patient to their GP on initial presentation in

344 the pharmacy, as well as following no symptom improvement. Acute pyelonephritis, chlamydial

345 urethritis and UTI in pregnancy were significantly less likely to be treated by a community

346 pharmacist than the other conditions presented.

348 In addition to findings suggesting a high level of appropriateness of antibiotic prescribing by

349 pharmacists $(334 / 380 ; 87.9 \%)$, this study also confirmed the existing literature indicating that

350 pharmacists are supportive of an expanded prescribing role for a limited range of infections and

351 antibiotics. $[19,21]$ In this regard, pharmacists suggested a stronger preference for prescribing

352 trimethoprim, amoxicillin and cefalexin and little support was suggested for prescribing

353 antibiotics such as rifampicin and ciprofloxacin. Pharmacists also indicated higher confidence in

354 the treatment of less complicated infections. This is consistent with findings from previous

355 Australian studies. [19-21] Notably, trimethoprim has been prescribed OTC in Scotland using a

356 strict protocol providing improved patient access. [6] 
358 In their responses to the case scenarios provided, most pharmacists would refer patients

359 following three days of no improvement on initial therapy (or following 24 hours in the CAP

360 case). This further supports the notion that community pharmacists are able to make decisions to

361 treat minor uncomplicated infections without treatment delays and appropriately refer to the GP

362 where complications arise. Minor ailments are reported to account for $10-20 \%$ of a doctors'

363 workload [31] with doctors showing support for the diversion of management of minor ailments

364 to non-medical prescribers including pharmacists. [32, 33]

365

366 In a majority $(426 / 630 ; 67.6 \%)$ of the vignette responses in this study, the pharmacist opted to

367 treat patients compared to referring them to the GP from the outset. As demonstrated in

368 international studies $[10,14]$, this level of responses to treat selected patient scenarios may

369 suggest that community pharmacists are willing to manage a range of self-limiting infections. In

370 addition, a large number of pharmacists followed the recommended guidelines leading to

371 appropriate antibiotic therapy and potentially reduced antibiotic resistance. [5] An advantage of

372 antibiotic management is immediate patient access. However, it is noteworthy that when

373 mupirocin was reclassified OTC in NZ an increased resistance occurred in part from it being

374 made available from pharmacies. [34] However, similar levels of resistance were recorded in

375 WA where it was only available on prescription. [34]

377 It was found that pharmacists in this study who estimated that less than three patients per week

378 would seek their treatment advice, were more likely to refer patients to the GP. A study in Spain

379 reported that the number of hours worked and high patient numbers were associated with 
380 generating more prescriptions by community pharmacists, concluding that longer work hours

381 was related to the pharmacist's understanding of their work. [35] This is consistent with the

382 present study where pharmacists from small turnover pharmacies were more likely to refer

383 patients to the GP compared to large turnover pharmacies. This may indicate a more

384 conservative approach to patient management than perceived by pharmacists in larger

385 pharmacies. A study of community pharmacists in WA revealed that pharmacies with a large

386 turnover were more supportive of the reclassification of chloramphenicol ophthalmic products

387 than small and medium sized pharmacies [13]. An explanation for this may be stronger

388 commercial interests for large pharmacies. Protocols would be essential to ensure antibiotic

389 stewardship if selected antibiotics were reclassified.

390

391 Older respondents ( $>51$ years) were less likely to refer patients to the GP and of those who

392 chose to treat the patient directly these older respondents were less likely to prescribe

393 appropriately. Caamaño at al. suggested that the more experienced pharmacists generated fewer

394 prescriptions and were more likely to refer a patient to their GP. [35] These findings are

395 supported by other studies where younger pharmacists were reported to place more importance

396 on the patient's and pharmacist's autonomy compared to older pharmacists, describing older

397 pharmacists as being 'more traditional' in their roles as pharmacists. [36, 37] Another

398 explanation may be the emphasis of education for older pharmacists would have been much less

399 patient centred. [38]

400

401 With a response rate of $37.5 \%$, it is likely that some non-responders may have views on

402 pharmacists' expanded prescribing of selected oral antibiotics which may differ from those based 
403 on the survey responders. However, with the high and consistent support for statements of views

404 on prescribing oral antibiotics, high level of pharmacists' willingness to treat the patients in 405 preference to GP referral, and overall high level of appropriateness of therapy selected, it is less 406 likely that non-respondents' responses would have significantly influenced the findings.

407 Furthermore, the wide range of conditions portrayed in the seven vignettes strengthens the 408 validity of the data. Case vignettes are often used to assess clinician's decision making behaviour 409 and judgements. [27] It should be highlighted that the literature suggests that this method 410 provides predictive behaviour in circumstances appropriated by the vignette, [27] thus the

411 vignettes provide a demonstration of potential performance. A limitation of the vignettes is that 412 although face and content validity of the vignettes were determined, the grading scale was not 413 specifically validated and therefore the reliability of the grading scale is not known.

415 This study demonstrated with the current framework of drug regulation that a "down-scheduling" 416 option could be utilised to enable improved access for the public to a limited range of antibiotics

417 for specific infections. This would also partially address the underutilisation of pharmacist's 418 skills and their high accessibility. Appropriate methods would need to be developed for this to 419 occur. [7]

420

421 Findings of this study should also be interpreted in context of the need to identify strategies and 422 protocols that minimise antibiotic misuse in the community. Potential self-diagnosis and self423 management of upper respiratory infections by the Australian community through use of 424 antibiotic repeat prescriptions is undesirable. [39] 


\section{Conclusion}

427 In general, pharmacists indicated a high level of appropriateness of antibiotic selection when

428 faced with a range of scenarios as vignettes. More complicated infections tended to be referred to

429 the doctor. The findings of this study warrant consideration by professional bodies regarding 430 expanding the role of pharmacists in the area of limited antibiotic prescribing for limited

431 infections, as one of the strategies to addressing antibiotic misuse in the community and reducing 432 unnecessary inconvenience and cost to the community. This would require the development of 433 prescribing protocols that ensured appropriate prescribing.

434

\section{Acknowledgments}

436 Authors wish to acknowledge all pharmacists who participated in this study, 437 
440

441

442

443

444

445

446

447

448

449

450

451

452

453

454

455

456

457

458

459

460

461

462

463

464

465

466

467

468

469

470

471

472

473

474

475

476

477

478

479

480

481

482

483

1. Gauld NJ, Kelly FS, Kurosawa N, Bryant LJ, Emmerton LM, Buetow SA. Widening consumer access to medicines through switching medicines to non-prescription: a six country comparison. PlosOne. 2014; 24:9(9):e107726.

2. Australian self-medication industry 2017 pre-budget submission http://www.asmi.com.au/media/46418/asmi_2017_budget_submission_-_final.pdf (accessed 2nd March, 2018)

3. Aronson JK. From prescription-only to over-the-counter medicine ('POM to P'): time for an immediate category. Br Med Bull. 2009; 90:63-9.

4. Gauld NJ, Zeng IS, Ikram RB, Thomas MG, Buetow SA. Antibiotic treatment of women with uncomplicated cystitis before and after allowing pharmacist-supply of trimethoprim. Int J Clin Pharm. 2017; 39(1): 165-172.

5. Huttner A, Harbarth S, Carlet J, Cosgrove S, Goossens G, Holmes A, Jarlier V, Voss A, Pittet D. Antimicrobial resistance: a global view from the 2013 World HealthcareAssociated Infections Forum. Antimicrob Resist Infect Control. 2013; 2:31.

6. Booth JL, Mullen AB, Thomson DA, Johnstone C, Galbraith SJ, Bryson SM, McGovern EM. Antibiotic treatment of urinary tract infection by community pharmacists: a cross sectional study. Br J Gen Pract. 2013; 63(609): e244-9.

7. Reeves DS, Finch RG, Bax RP, Davey PG, Po AL, Lingam G, Mann SG, Pringle MA. Self-medication of antibacterials without prescription (also called 'over-the-counter' use). A report of a working party of the British Society for Antimicrobial Chemotherapy. J Antimicrob Chemother.1999; 44(2): 163-177.

8. Weller TMA, Jamieson CE. The expanding role of the antibiotic pharmacist. J Antimicrob Chemother. 2004; 54(2):295-298.

9. Klepser ME, Adams AJ, Klepser DG. Antimicrobial stewardship in outpatient settings: leveraging innovative physician-pharmacist collaborations to reduce antibiotic resistance. Health Secur. 2015; 13(3): 166-73.

10. Tonna AP, Stewart DC, West B, McCaig DJ. Exploring pharmacists' perceptions of the feasibility and value of pharmacist prescribing of antimicrobials in secondary care in Scotland. IJPP. 2010; 18(5):312-319.

11. Hale A, Coombes I, Stokes J, Aitken S, Clark F, Nissen L. Patient satisfaction from two studies of collaborative doctor - pharmacist prescribing in Australia. Health Expect. 2016; 19(1):49-61.

12. Samsom LN, ed. Australian pharmaceutical formulary and handbook. $24^{\text {th }}$ edn. Canberra: Pharmaceutical Society of Australia; 2018.

13. Alkhatib L, Parsons R, Czarniak P, Sunderland VB. An evaluation of the reclassification of ophthalmic chloramphenicol for the management of acute bacterial conjunctivitis in community pharmacies in Western Australia. IJPP. 2015; 23(2):111-120.

14. Law MR, Ma T, Fisher J, Sketris IS. Independent pharmacist prescribing in Canada. Can Pharm J. 2012; 145(1):17-23.

15. Lynas K. Pharmacist Prescribing Takes Effect in Alberta. Can Pharm J. 2007; 140(3):150-150.

16. Zellmer WA. Collaborative drug therapy management. AJHP. 1995; 52(15):1732.

17. Tonna AP, Stewart D, West B, McCaig D. Pharmacist prescribing in the UK - a literature review of current practice and research. J Clin Pharm Ther. 2007; 32(6): 545-556. 
484

485

486

487

488

489

490

491

492

493

494

495

496

497

498

499

500

501

502

503

504

505

506

507

508

509

510

511

512

513

514

515

516

517

518

519

520

521

522

523

524

525

526

527

18. Hughes CA, Makowsky M, Sadowski CA, Schindel TJ, Yuksel N, Guirguis LM. What prescribing means to pharmacists: a qualitative exploration of practising pharmacists in Alberta. IJPP. 2014; 22(4):283-291.

19. Hoti K, Sunderland B, Hughes J, Parsons R. An evaluation of Australian pharmacist's attitudes on expanding their prescribing role. Pharm World Sci. 2010; 32(5):610-621.

20. Kay OC, Bajorek BV, Brien J-AE. Pharmacist prescribing activities - An electronic survey on the opinions of Australian Pharmacists. J Pharm Pract Res. 2006; 36(3):199203.

21. Hoti K, Hughes J; Sunderland B. Identifying the perceived training needs for Australian pharmacist prescribers. IJPP.2014; 22:38-46.

22. Hoti K, Hughes J, Sunderland B. Pharmacy clients' attitudes to expanded pharmacist prescribing and the role of agency theory on involved stakeholders. IJPP. 2011; 19(1):512.

23. Paudyal V, Hansford D, Cunningham S, Stewart D. Over-the-counter prescribing and pharmacists' adoption of new medicines: diffusion of innovations. RSAP. 2013;9(3):251262.

24. Paudyal V, Hansford D, Cunningham S, Stewart D. Pharmacists' perceived integration into practice of over-the-counter simvastatin five years post reclassification. Int J Clin Pharm. 2012; 34(5):733-8.

25. Zayegh I, Charrois T, Hughes J, Hoti K. Antibiotic repeat prescriptions: are patients notrefilling them properly? JoPPP. 2014; 7:17

26. Res R, Hoti K, Charrois T. Pharmacists' perceptions regarding optimization of antibiotic prescribing in the community. J Pharm Pract; 2017; 30(2):146-153.

27. Evans SC, Roberts MC, Keeley JW, Blossom JB, Amaro CM, Garcia AM, Odar Stough C, Canter KS, Robles R, Reed GM. Vignette methodologies for studying clinician's decision making: Validity, utility, and application in ICD-11 field studies. IJCHP. 2015; 15(2): 160-170.

28. Atzmüller C. Steiner CM. Experimental vignette studies in survey research. Methodology (Gott). 2010; 6:128-138.

29. Antibiotic Expert Groups. Therapeutic guidelines: Antibiotic. Version 15. Melbourne: Therapeutic Guidelines; 2014.

30. Tabachnick BG. Using multivariate statistics / Barbara G. Tabachnick, Linda S. Fidel. 6th ed.. ed. Boston: Boston : Pearson Education; 2013.

31. Banks I. Self care of minor ailments: a survey of consumer and healthcare professional beliefs and behaviour. SelfCare. 2010; 2010(1):1-13.

32. Mansell K, Bootsman N, Kuntz A, Taylor J. Evaluating pharmacist prescribing for minor ailments. IJPP. 2015; 23(2):95-101.

33. Bayliss E, Rutter P. General practitioners' views on recent and proposed medicine switches from POM to P. Pharm J. 2004; 273(7328):819-821.

34. Upton A, Lang S, Heffernan H. Mupirocin and Staphylococcus aureus: a recent paradigm of emerging antibiotic resistance. J Antimicrob Chemother. 2003; 51(3): 613-7.

35. Caamaño F, Tomé-otero M, Takkouche B, Gestal-otero JJ. Factors associated with the decision of Spanish pharmacists to prescribe medicines and to recommend the visit to a doctor. Pharmacoepidemiol Drug Saf. 2004; 13(11):817-820. 
528

529

530

531

532

533

534

535

536

36. Pendergast JF, Kimberlin CL, Berardo DH, McKenzie LC. Role orientation and community pharmacists' participation in a project to improve patient care. Soc Sci Med. $1995 ; 40(4): 557-565$.

37. Isorna F, Tome-Otero M, Takkouche B, Figueiras A. Factors related with prescription requirement to dispense in Spain. Pharmacoepidemiol. Drug Saf. 2004; 13(6):405-409.

38. McWhinney A. A history of pharmacy in Western Australia. Perth: Pharmaceutical Society of Western Australia. 1975.

39. Newby DA, Fryer JL, Henry DA. Effect of computerised prescribing on use of antibiotics. Med J Aust. 2003; 178(5):210-3. 


\section{Table $\mathbf{1}$ (on next page)}

Demographic profile of the survey respondents $(n=90)$, and their association with support for rescheduling of oral antibiotics.

P-values were obtained from the Chi-square test unless otherwise specified. 
1 Table 1: Demographic profile of the survey respondents $(\mathrm{n}=90)$, and their association with

2 support for rescheduling of oral antibiotics. P-values were obtained from the Chi-square test unless 3 otherwise specified.

\begin{tabular}{|c|c|c|c|}
\hline Variable n (\%) & $\begin{array}{c}\text { Number } \\
(\%)\end{array}$ & $\begin{array}{c}\text { Number }(\%) \text { supporting } \\
\text { rescheduling }\end{array}$ & p-value \\
\hline Gender $(n=90)$ & & & 0.8504 \\
\hline Female & $44(48.9)$ & $24(54.6)$ & \\
\hline Male & $46(51.1)$ & $26(56.5)$ & \\
\hline $\operatorname{Age} \operatorname{group}(\mathrm{n}=89)$ & & & 0.9166 \\
\hline $21-30$ & $31(34.8)$ & $17(54.8)$ & \\
\hline $31-40$ & $32(36.0)$ & $19(59.4)$ & \\
\hline $41-50$ & $10(11.2)$ & $5(50.0)$ & \\
\hline 51 or more & $16(18.0)$ & $8(50.0)$ & \\
\hline Years registered as pharmacist $(\mathrm{n}=88)$ & & & 0.5045 \\
\hline 1 to 5 & $32(36.4)$ & $17(53.1)$ & \\
\hline 6 to 20 & $35(39.8)$ & $22(62.9)$ & \\
\hline 21 or more & $21(23.9)$ & $10(47.6)$ & \\
\hline Years worked in a pharmacy $(\mathrm{n}=90)$ & & & 0.9149 \\
\hline 1 to 5 & $25(27.8)$ & $13(52.0)$ & \\
\hline 6 to 20 & $44(48.9)$ & $25(56.8)$ & \\
\hline 21 or more & $21(23.3)$ & $12(57.1)$ & \\
\hline \multicolumn{4}{|l|}{ Position held in pharmacy* $(n=93)$} \\
\hline Proprietor & $31(34.4)$ & $18(58.1)$ & 0.7284 \\
\hline Manager & $28(31.1)$ & $16(57.1)$ & 0.8386 \\
\hline Pharmacist in charge & $23(25.6)$ & $13(56.5)$ & 0.9139 \\
\hline Employed pharmacist & $6(6.7)$ & $3(50.0)$ & $1.0^{\wedge}$ \\
\hline Consultant pharmacist & $3(3.3)$ & $2(66.7)$ & $1.0^{\wedge}$ \\
\hline Other position & $2(2.2)$ & 0 & 0.1948 \\
\hline \multicolumn{4}{|l|}{ Pharmacy setting§ $(n=90)$} \\
\hline Group of shops & $24(26.7)$ & $14(58.3)$ & 0.7491 \\
\hline City & $2(2.2)$ & $2(100)$ & $0.5006^{\wedge}$ \\
\hline Neighbourhood & $23(25.6)$ & $11(47.8)$ & 0.3872 \\
\hline Stand-alone & $4(4.4)$ & $2(50.0)$ & $1.0^{\wedge}$ \\
\hline Next to doctor's surgery & $14(15.6)$ & $9(64.3)$ & 0.4744 \\
\hline Regional shopping centre & $12(13.3)$ & $7(58.3)$ & 0.8352 \\
\hline Medical centre & $10(11.1)$ & $4(40.0)$ & 0.3299 \\
\hline Other setting & $1(1.1)$ & $1(100)$ & $1.0^{\wedge}$ \\
\hline Counselling room available & $80(88.9)$ & $45(56.3)$ & $0.7461^{\wedge}$ \\
\hline Forward Dispensing area & $38(42.2)$ & $24(63.2)$ & 0.2147 \\
\hline Operation size (turn-over) ${ }^{\mathbb{d}}(\mathrm{n}=89)$ & & & 0.5137 \\
\hline Small $(<\$ 1 \mathrm{M})$ & $26(29.2)$ & $13(50.0)$ & \\
\hline Medium (\$1M-\$2M) & $38(42.7)$ & $24(63.2)$ & \\
\hline Large $(\$ 2 \mathrm{M}+)$ & $25(28.1)$ & $13(52.0)$ & \\
\hline
\end{tabular}


Pharmacy location $(\mathrm{n}=90)$

Metropolitan $67(74.4)$

$23(25.6)$

$37(55.2)$

Rural

$13(56.5)$

$4{ }^{*}$ There were2 respondents who classified themselves as consultant and proprietor pharmacists, and 1 as consultant

5 and pharmacist in charge

6 \& 1 missing response

$7 \wedge$ Fisher's Exact Test

8 \& 2 espondents may select 1 or more settings for their pharmacy 


\section{Table 2 (on next page)}

Respondents' characteristics associated with their decision to refer a patient to their general practitioner (GP) initially ( $n=630$; results from the Generalised Estimating Equation model). 
1 Table 2: Respondents' characteristics associated with their decision to refer a patient to their 2 general practitioner (GP) initially $(n=630$; results from the Generalised Estimating Equation 3 model).

4

\begin{tabular}{|c|c|c|c|c|}
\hline Variable & $\begin{array}{l}\text { Number (\%) } \\
\text { referring to } \\
\text { general } \\
\text { practitioner }\end{array}$ & $\begin{array}{c}\text { Adjusted } \\
\text { Odds Ratio }\end{array}$ & $95 \% \mathrm{CI}$ & p-value \\
\hline \multicolumn{5}{|l|}{ Case type } \\
\hline Otitis media & $13 / 90(14.4)$ & 0.13 & $0.06-0.27$ & $<.0001$ \\
\hline UTI in pregnancy & $37 / 90(41.1)$ & 0.64 & $0.35-1.14$ & 0.1279 \\
\hline CAP & $32 / 90(35.6)$ & 0.49 & $0.27-0.88$ & 0.0168 \\
\hline Tonsillitis & $10 / 90(11.1)$ & 0.1 & $0.05-0.20$ & $<.0001$ \\
\hline Chlamydial urethritis & $45 / 90(50.0)$ & 0.95 & $0.57-1.58$ & 0.8479 \\
\hline Mid early cellulitis & $21 / 90(23.3)$ & 0.25 & $0.13-0.47$ & $<.0001$ \\
\hline Acute pyelonephritis & $46 / 90(51.1)$ & 1 (reference) & & \\
\hline \multicolumn{5}{|l|}{ Gender } \\
\hline Female & $96 / 308(31.2)$ & 0.53 & $0.32-0.91$ & 0.0198 \\
\hline Male & $108 / 322(33.5)$ & 1 (reference) & & \\
\hline \multicolumn{5}{|l|}{ Age group } \\
\hline 51 or more & $23 / 112(20.5)$ & 0.38 & $0.18-0.84$ & 0.0165 \\
\hline $\mathrm{Up}$ to 50 & $181 / 518(34.9)$ & 1 (reference) & & \\
\hline \multicolumn{5}{|l|}{ Setting of pharmacy } \\
\hline Medical centre & $34 / 70(48.6)$ & 2.29 & $1.19-4.43$ & 0.0137 \\
\hline Other & $170 / 560(30.4)$ & 1 (reference) & & \\
\hline \multicolumn{5}{|c|}{ Size of pharmacy (turnover) } \\
\hline Small $(<\$ 1 \mathrm{M})$ & $71 / 182(39.0)$ & 2.38 & $1.21-4.69$ & 0.0122 \\
\hline Medium $(\$ 1 M-\$ 2 M)$ & $88 / 266(33.1)$ & 1.83 & $0.92-3.63$ & 0.0837 \\
\hline Large $(>\$ 2 \mathrm{M})$ & $41 / 175(23.4)$ & 1 (reference) & & \\
\hline \multicolumn{5}{|l|}{ Patients* } \\
\hline Up to 3 per week & $33 / 63(52.4)$ & 2.86 & $1.18-6.95$ & 0.0205 \\
\hline 4 or more per week & $171 / 567(30.2)$ & 1 (reference) & & \\
\hline \multicolumn{5}{|l|}{ Rescheduling } \\
\hline Neutral/Disagree & $113 / 280(40.4)$ & 1.97 & $1.16-3.33$ & 0.0116 \\
\hline Agree & $91 / 350(26.0)$ & 1 (reference) & & \\
\hline
\end{tabular}

*The estimated number of patients per week at pharmacy who would better be treated with oral antibiotics. Numbers are the number $n$ of respondents and the percentage in parentheses 


\section{Table 3 (on next page)}

Variables associated with appropriateness of therapy selected ( $n=426$; results from the Generalised Estimating Equation model). 
1 Table 3: Variables associated with appropriateness of therapy selected ( $n=426$; results from the

2 Generalised Estimating Equation model).

\begin{tabular}{|c|c|c|c|c|}
\hline Variable & Number correct $(\%)$ & Odds Ratio & $95 \% \mathrm{CI}$ & p-value \\
\hline \multicolumn{5}{|l|}{ Case type } \\
\hline Otitis media & $59 / 77(76.6)$ & 0.17 & $0.05-0.60$ & 0.006 \\
\hline UTI in pregnancy & $8 / 53(15.1)$ & 0.01 & $0.00-0.04$ & $<.0001$ \\
\hline CAP & $46 / 58(79.3)$ & 0.21 & $0.05-0.85$ & 0.0283 \\
\hline Tonsillitis & $74 / 80(92.5)$ & 0.62 & $0.15-2.58$ & 0.5146 \\
\hline Chlamydial urethritis & $39 / 45(86.7)$ & 0.37 & $0.10-1.36$ & 0.1337 \\
\hline Mid early cellulitis & $66 / 69(95.7)$ & 1.16 & $0.23-5.83$ & 0.8528 \\
\hline Acute pyelonephritis & $42 / 44(95.5)$ & 1 (reference) & & \\
\hline \multicolumn{5}{|l|}{ Age group } \\
\hline 51 or more & $63 / 89(70.8)$ & 0.44 & $0.22-0.88$ & 0.02 \\
\hline Up to 50 & 266/337 (78.9) & 1 (reference) & & \\
\hline \multicolumn{5}{|l|}{ Work position } \\
\hline Consultant pharmacist & $9 / 13(69.2)$ & 0.32 & $0.14-0.73$ & 0.0068 \\
\hline Other & $320 / 413(77.5)$ & 1 (reference) & & \\
\hline
\end{tabular}

3

4

5

6

7 


\section{Figure 1}

\section{Respondents' level of support for statements of views on down scheduling of oral antibiotics $(n=90)$}

OTC prescribing of oral antibiotics is essential for a limited range of infections

Selected oral antibiotics should be down scheduled from S4 to S3

Provision of OTC oral antibiotics can increase resistance to antibiotics

The provision of OTC oral antibiotics helps ease the workload on doctors

Current design of pharmacy would allow diagnosis of infections and supply of oral antibiotics

The availability of selected OTC oral antibiotics increases patient's easy access to antibiotics

OTC prescribing of selected oral antibiotics offers better use of pharmacists' knowledge and skills

The capability to prescribe selected OTC oral antibiotics ensures that patients can be treated in a timely manner

The provision of selected OTC oral antibiotics increases the recognition of pharmacists' knowledge and skills by pharmacy clients 


\section{Figure 2}

Respondents' level of support for 'down scheduling' of specific oral antibiotics $(n=90)$

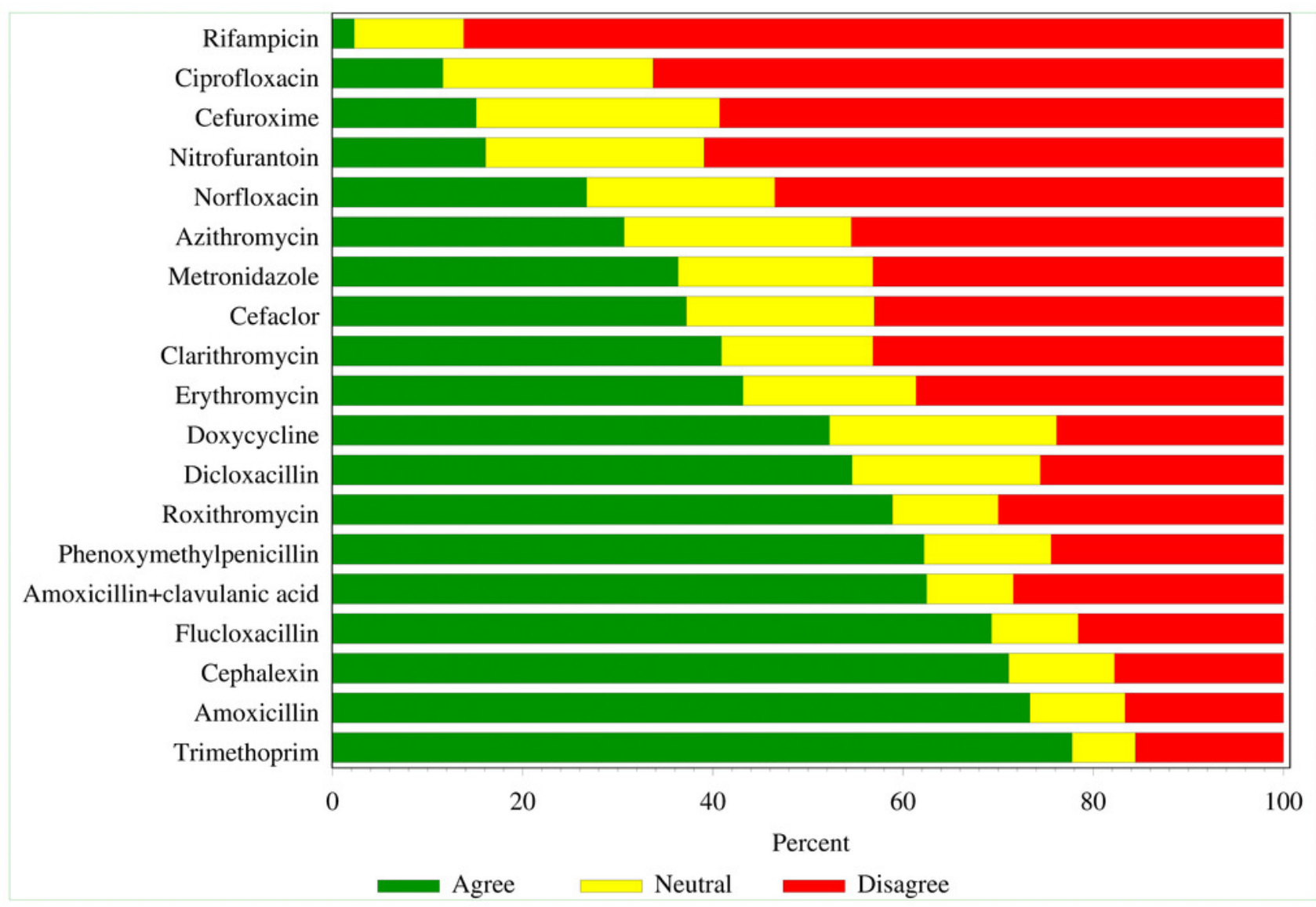




\section{Figure 3}

Summary of respondents' choice to refer a patient to a general practitioner (GP) initially rather than treat with an oral antibiotic and the appropriateness of antibiotic selected $(n=426)$

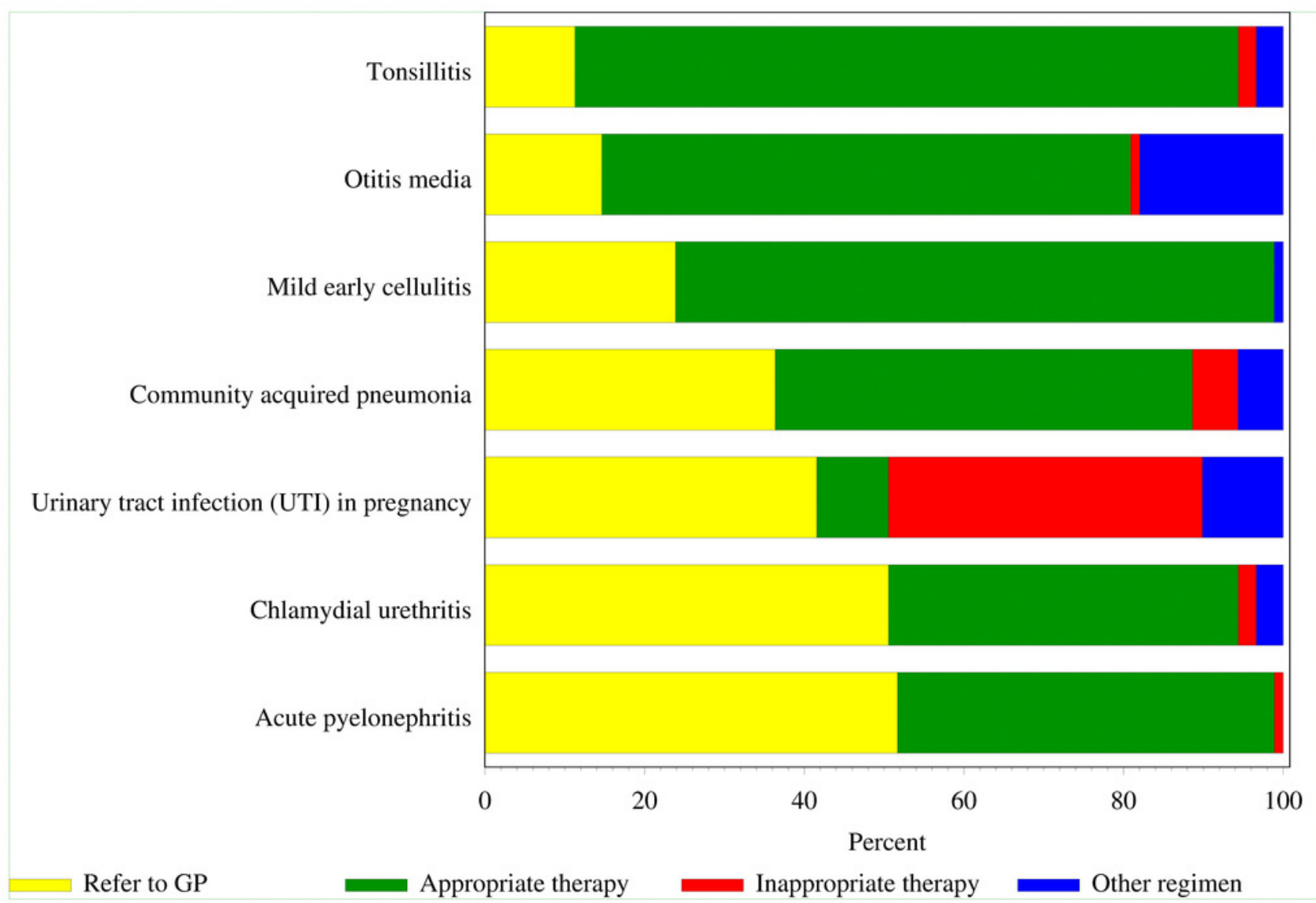




\section{Figure 4}

Respondents' level of support for therapy following 3 days (or 24 hours for community acquired pneumonia) of no improvement on initial therapy $(n=426)$.

(Note: * 'Other' includes both drug and non-drug therapies).

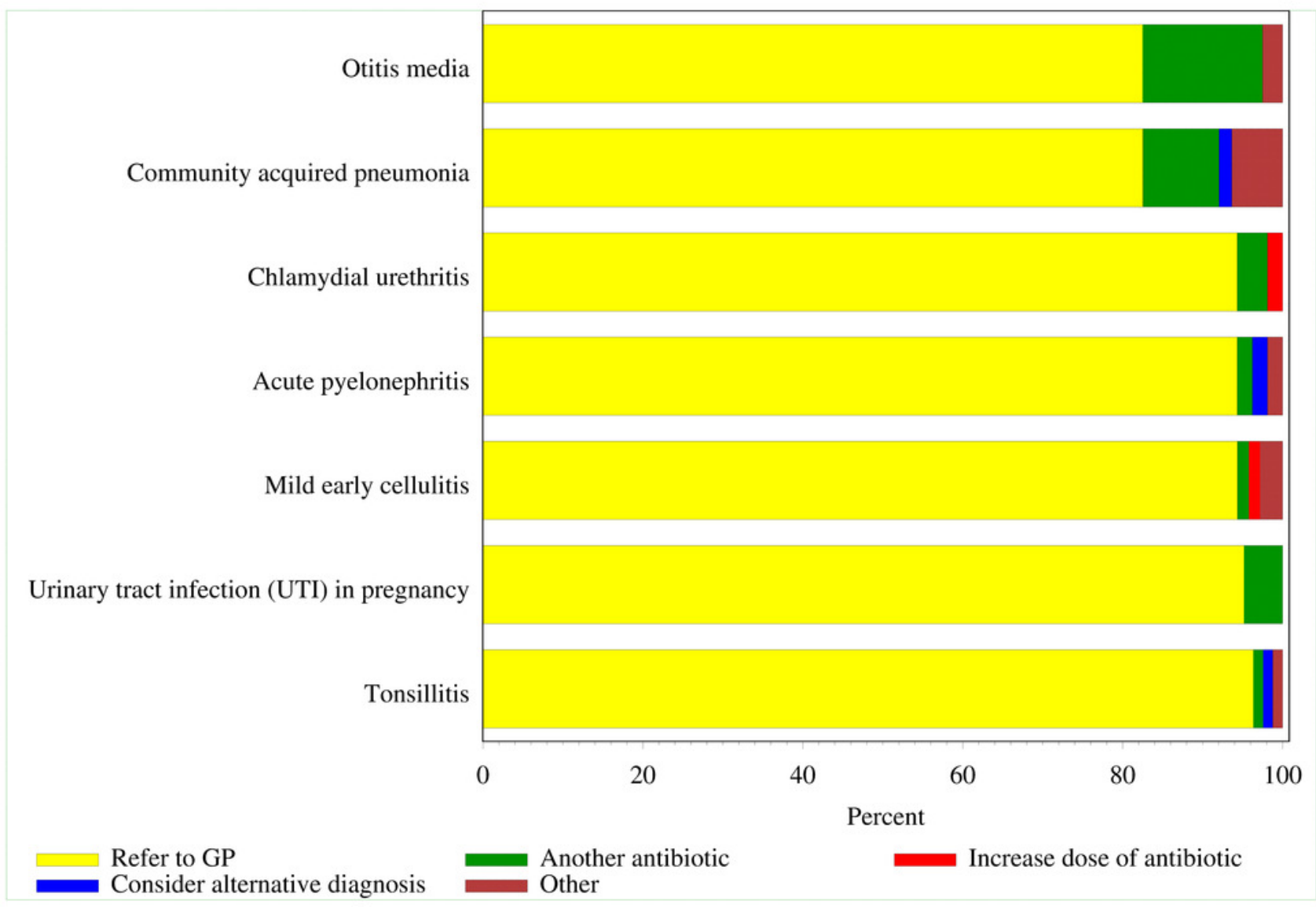

